

\title{
A multicenter analysis of the role of prophylactic transfusion of blood products in patients with cirrhosis and esophageal varices undergoing endoscopic band ligation
}

\author{
Annabel Blasi, ${ }^{1,7,9, \dagger}$ Salvador Machlab, ${ }^{2, \dagger}$ Raquel Risco, ${ }^{1}$ Joao Pedro Costa-Freixas, ${ }^{2}$ Geovanny Hernández-Cely, ${ }^{3,5}$ \\ Diana Horta, ${ }^{4}$ Alex Bofill, ${ }^{5}$ Pablo Ruiz-Ramirez, ${ }^{4}$ Joaquim Profitos, ${ }^{6}$ Josep Marti Sanahuja, ${ }^{1,9}$ \\ Alejandro Fernandez-Simon, ${ }^{5}$ Mercedes Vergara Gómez, ${ }^{2,7}$ Jordi Sánchez-Delgado, ${ }^{2,7}$ Andrés Cardenas ${ }^{7,8,9, *}$

\begin{abstract}
${ }^{1}$ Anesthesia Department, Hospital Clinic, Barcelona, Spain; ${ }^{2}$ Digestive Diseases Department, Corporació Sanitària Universitària Parc Taulí, Institut d Investigació i Innovació Parc Taulí I3PT, Universitat Autònoma de Barcelona, Spain; ${ }^{3}$ Fundación Cardioinfantil, Gastroenterology and Hepatology Department, Colombia; ${ }^{4}$ Gastroenterology Department, Hospital Universitario Mutua de Terrassa, Spain; ${ }^{5}$ GI Unit, Institut de Malalties Digestives $i$ Metaboliques, Hospital Clinic, Barcelona, Spain; ${ }^{6}$ Gastroenterology Department, Consorci Sanitari de Terrassa, Spain; ${ }^{7}$ Ciber de Enfermedades Hepáticas y Digestivas (CIBEREHD), Spain; ${ }^{8}$ GI E Liver Transplant Unit, Institut de Malalties Digestives i Metaboliques, Hospital Clinic, Barcelona, Spain; ${ }^{9}$ Institut d'Investigacions Biomèdiques August Pi-Sunyer (IDIBAPS), Barcelona Spain
\end{abstract}

JHEP Reports 2021. https://doi.org/10.1016/j.jhepr.2021.100363

Background \& Aims: Prophylactic administration of platelets and fresh frozen plasma (FFP) has been recommended in patients with cirrhosis with low platelets and/or prolonged international normalized ratio (INR) without scientific evidence to support this practice. In this analysis, we evaluated the use of prophylactic administration of blood products in outpatients with cirrhosis undergoing endoscopic band ligation (EBL).

Methods: This is a multicenter retrospective analysis of consecutive EBL procedures in patients with cirrhosis at 4 hospitals in Spain from 01/2010-01/2017. FFP and/or platelet transfusion were given at the discretion of the physician if INR was $>1.5$ and/ or platelet count $<50 \times 10^{9} / \mathrm{L}$. Patient demographics, endoscopic findings, bleeding events after EBL, and the use of prophylactic FFP or platelets were recorded.

Results: A total of 536 patients underwent 1,472 EBL procedures: 72\% male; main etiology HCV and alcohol (72\%); median MELD score 11; Child-Pugh A/B/C (59/33/8\%). EBL procedures were performed for primary (51\%) or secondary (49\%) prophylaxis. A median of 2 procedures per patient were performed. ${ }^{1-4}$ FFP and/or platelets were administered in 41 patients (7.6\%). The prophylactic transfusion protocol was followed in $16 \%$ and $28 \%$ of procedures with high INR and/or low platelets, respectively. Post-EBL bleeding occurred in 26 out of 536 patients (4.8\%) and in 33 out of 1,472 procedures (2.2\%). Bleeding was due to post-EBL ulcers in 21 patients and due to band dislodgment in 5. In 6 patients, bleeding occurred within 24 hours and in the remaining patients it occurred within 2 weeks after EBL. In those that bled, 7 met criteria for transfusion (2 for FFP and 5 for platelets), of whom only 1 received FFP and 4 received platelets; the remaining 19 patients did not meet criteria for transfusion. There was no association between INR or platelet count and bleeding events. Univariate and multivariate analysis revealed that Child-Pugh and MELD scores were risk factors for post-EBL bleeding.

Conclusions: The incidence of post-EBL bleeding is low and is associated with advanced liver disease. Post-EBL bleeding was not related to baseline INR/platelet count and most outpatients with post-EBL bleeding did not meet criteria for prophylactic transfusion.

Lay summary: Patients with chronic liver disease or cirrhosis and enlarged veins (varices) of the esophagus that can potentially bleed commonly need an endoscopy to treat these varices with elastic rubber bands (endoscopic band ligation). Some patients have low platelet counts or prolonged coagulation tests. This analysis of 4 centers evaluated the use of prophylactic administration of blood products in outpatients with cirrhosis undergoing endoscopic band ligation. The results showed that bleeding after band ligation is uncommon and that if bleeding occurs it does not seem to be related with coagulation tests or the administration of blood products to prevent bleeding after band ligation of esophageal varices.

(c) 2021 The Authors. Published by Elsevier B.V. on behalf of European Association for the Study of the Liver (EASL). This is an open access article under the CC BY-NC-ND license (http://creativecommons.org/licenses/by-nc-nd/4.0/).

\footnotetext{
Keywords: acute variceal bleeding; cirrhosis; MELD score; Child Score; endoscopic band ligation; esophageal varices; primary prophylaxis; secondary prophylaxis; post-band ligation ulcer; gastrointestinal bleeding; fresh frozen plasma; platelets; platelet transfusion.

Received 7 May 2021; received in revised form 30 August 2021; accepted 1 September 2021; available online 16 September 2021

$\dagger$ Both authors collaborated equally to this study.

* Corresponding author. Address: Institute of Digestive Diseases \& Metabolism, Hospital Clinic of Barcelona, University of Barcelona, Villarroel 170, Esc 3-2, 08036 Barcelona, Spain; Tel.: (+34) 93227 5513, fax: (+34) 932279850

E-mail addresses: acardena@clinic.cat, acv69@hotmail.com (A. Cardenas).
} 


\section{Introduction}

Variceal hemorrhage is a major cause of morbidity and mortality in patients with cirrhosis. It occurs in up to $25-40 \%$ of patients with cirrhosis and each episode is associated with up to a $20 \%$ risk of mortality, with an increased risk of repeated episodes of bleeding if no prevention measures are provided. ${ }^{1,2}$ Endoscopic band ligation (EBL) is considered a first-line therapy for the control of acute variceal bleeding. In addition, EBL along with beta-blockers is always recommended as a measure of secondary prophylaxis of variceal bleeding. EBL is also commonly used as primary prophylaxis of variceal bleeding when beta-blockers are contraindicated or not tolerated. ${ }^{2,3}$ A serious complication of this procedure is major bleeding due to post-ligation ulcer, which usually occurs 1 to 2 weeks after the procedure. Published data indicates that the incidence of this complication ranges between $1.5 \%-10 \%{ }^{4-8}$ In most cases post-EBL ulcer bleeding requires hospitalization and blood transfusions; mortality rates in this setting can be as high as $50 \%{ }^{9}$

Advanced liver disease with high Child-Pugh and model for end-stage liver disease (MELD) score and presence of ascites are described risk factors for post-EBL ulcer bleeding. ${ }^{4-8}$ Bleeding risk assessment is a well-established practice in the clinical evaluation of patients with liver disease. However, none of the commonly used tests are considered reliable for predicting bleeding risk in patients with cirrhosis that undergo EBL. Conventional tests, such the prothrombin time or international normalized ratio for prothrombin time (INR) and activated partial thromboplastin time do not correlate with procedure-related bleeding in patients with cirrhosis. ${ }^{10}$ Extremely low platelet counts (between 5,000-10,000/ $\mu \mathrm{l}$ ) may correlate with the risk of bleeding; however, there is limited data to support this contention. ${ }^{11}$ Although not widespread, some centers use preprocedure cut-offs to determine whether to administer fresh frozen plasma (FFP) and platelets before an EBL in patients with cirrhosis with an INR $\geq 1.5$ and platelet count $\leq 50,000 / \mu$ l. These cut-offs are empirically used but there is no evidence-based rationale for this practice given that the use of blood products to reach a normal coagulation value does not seem to be associated with less bleeding complications in patients with cirrhosis. ${ }^{10}$ In addition, the vast majority of bleeding episodes occur $>24$ hours after the procedure rendering the use of blood products of limited value for the prevention of bleeding. There are no established methods of preventing post-EBL ulcer bleeding and FFP and platelet transfusion do not seem to prevent bleeding episodes and confer a benefit in these patients.

Since transfusions of FFP and platelets may increase the risk of adverse events (infection, anaphylactic reaction, etc) and even the risk of bleeding due to portal pressure elevation from excessive volume expansion with plasma this practice has been called into question by several publications..$^{10,12-14}$ Therefore, the aim of this study was to analyze the prevalence and characteristics of post-EBL ulcer bleeding and examine the role of prophylactic blood product transfusion in this setting.

\section{Patients and methods}

We retrospectively reviewed the medical records of consecutive outpatients with cirrhosis who underwent a scheduled EBL for primary and secondary prophylaxis from $01 / 2010-01 / 2017$ at 4 centers in Spain: Hospital Clinic, Barcelona, Universitari Mutua de Terrassa, Consorci Sanitari de Terrassa and Corporació Sanitària Parc Taulí. Patients with EBL and active variceal bleeding were excluded. Approval to carry out this study was granted by the Institutional Review Board of all the participating centers.

Varices were classified as small or large according to AASLD guidelines. ${ }^{1}$ The EBL procedure was performed in standard fashion as described elsewhere. ${ }^{3}$ The banding procedure was performed by experienced endoscopists during withdrawal of the endoscope and after aspiration of air and fluids of the stomach. The bands were placed with the same device ( 4 or 6 Shooter Universal Saeed MultiBand Ligator, Cook Medical, or Speedband Superview Super 7, Multiple Band Ligator Kit Boston Scientific). The endoscopes used were adult gastroscope type Olympus GIF 160 (Olympus Medical Systems, Hamburg, Germany).

Following EBL, proton pump inhibitors (PPIs) were administered for at least 7 days in all patients. Patients received sedation with propofol using a target-controlled infusion system (FreseniusVial; Chemin de Fer, Beziers, France) or a titrated dose of propofol. Patients with primary prophylaxis did not receive nonselective beta-blockers, whereas those undergoing secondary prophylaxis were administered non-selective beta-blockers (propranolol, nadolol, or carvedilol) at the discretion of the treating physician. Patients were observed for at least 4-6 hours after the procedure. Food intake was allowed 6-12 hours following the endoscopic procedure. Standard blood tests and coagulation assays were assessed in all patients before procedure. The recommended transfusion protocol was the same in all centers and given at the physician's discretion. A transfusion of FFP ( $15 \mathrm{ml} \mathrm{x} \mathrm{kg}$ ) was administered if INR was $\geq 1.5$ and 1 pool of platelets was administered if the platelet count was $\leq 50 \times 10^{9} / \mathrm{L}$.

Protocol compliance was defined as blood product transfusion depending on the coagulation values. Bleeding was defined as any episode of fresh hematemesis $>2$ hours after EBL, drop in hemoglobin levels $>2 \mathrm{~g} / \mathrm{L}$, and/or melena. ${ }^{15}$ It was considered early if it occurred within 24 hours and as late if it occurred after 24 hours. A severe complication was defined as hemodynamic instability or blood product requirement. Patients were followed for 4 weeks after the procedure. Post-EBL bleeding was managed as an episode of acute variceal bleeding according to guidelines. ${ }^{1,2,15}$

\section{Statistical analysis}

The main analysis was performed per patient. However, analysis per procedures was also performed. Analysis of continuous variables was expressed as median and interquartile range. The chisquare test or the Fisher's exact test was used for qualitative or dichotomized variables, and the Mann-Whitney test for continuous variables. Variables with statistical significance $(p<0.05)$ were selected for inclusion in a logistic regression model. A 2tailed $p<0.05$ was taken as representing statistical significance. All analyses were performed with the SPSS version 25 software (Chicago, IL).

\section{Results}

A total of 536 patients underwent 1,472 EBL sessions between 2010 and 2017. The number of patients included by every institution was as follows: Hospital Clinic of Barcelona 401, Hospital Universitario Mutua de Terrassa 56, Consorcio Sanitario de Terrassa 25, and Corporación Sanitària Parc Taulí 54 patients. The median procedure per patient was $2 .^{1-4}$ The baseline characteristics are described in Table 1. Primary prophylaxis was the indication in $51 \%$ of procedures, and secondary prophylaxis in $49 \%$. 
Table 1. Baseline characteristics of 536 patients that underwent 1,472 EBL procedures.

\begin{tabular}{lr}
\hline Age (years) & $61(52-70)$ \\
Sex (M/F) & $384 / 152$ \\
Child score & $6(5-8)$ \\
Child class A, B, C (\%) & $59 / 33 / 8$ \\
MELD & $11(8-14)$ \\
Etiology: HCV, alcohol, other (\%) & $(40,31,29)$ \\
\hline Primary prophylaxis/Secondary prophylaxis (\%) & $51 / 49$ \\
Creatinine (mg/dl) & $0.8(0.7-1.1)$ \\
Bilirubin (mg/dl) & $1.3(0.8-2.0)$ \\
INR & $1.26(1.15-1.42)$ \\
Platelets x 109/L & $91(60-126)$ \\
APTT (seconds) & $31(28-34)$ \\
Fibrinogen (g/L) & $2.6(2-3.6)$ \\
Transfusion pre-procedure n (\%) & \\
Any blood product & $37(6.9)$ \\
FFP & $18(3.4)$ \\
Platelets & $24(4.5)$ \\
Bleeding n (\%) & $26(4.8)$ \\
$\quad$ Early (within 24 hours) & $5(0.9)$ \\
Late (within 15 days) & $21(3.9)$ \\
\hline
\end{tabular}

APTT, activated partial thromboplastin time; EBL, endoscopic band ligation; FFP, fresh frozen plasma; INR, international normalized ratio; MELD, model for end-stage liver disease.

Twenty-six patients (4.8\%) experienced bleeding after EBL: bleeding was early in 6 patients (1.1\%) and late in 20 (3.5\%). An analysis per procedure showed that bleeding occurred in $33 /$ $1,472(2.2 \%)$. Bleeding was due to post-EBL ulcers in 21 patients (late) and due to band dislodgment in 5 (early complications). There was no association of post-EBL bleeding with the first or last procedure in a sequence of bandings.

As described in Fig. 1, a total of 108 patients had INR $\geq 1.5$ and 85 patients had a platelet count $\leq 50,000 / \mu l$. FFP was administered to 18 patients (3.4\%) and platelets were administered in 24 patients (4.5\%); 2 patients received both FFP and platelets. Only $16 \%(17 / 108)$ of those that met criteria were transfused with FFP and $28 \%(24 / 85)$ of those that met criteria received platelet transfusion. A small proportion of those with an elevated INR $(2.7 \% ; n=3)$ and low platelets $(7 \% ; n=6)$ had post-EBL bleeding. In those that bled after EBL, only 7 met criteria for transfusion (2 for FFP and 5 for platelets). Of these, 1 with elevated INR received FFP and 4 received platelet transfusion. Of note, 8 patients out of 108 had an INR >2.5; only one of them underwent transfusion and bled. Fifteen patients out of 108 had a platelet count
$<30,000$; none of them bled. Of the 26 patients that bled after EBL, 19 did not meet criteria for transfusion.

We performed a univariate analysis per patient and per procedure to determine the relationship between bleeding and transfusion. As shown in Table 2, the univariate analysis per patient showed that MELD, Child-Pugh score and transfusion were risk factors for post-EBL bleeding. However, the multivariate analysis did not reveal that Child-Pugh class, MELD score, prophylactic transfusion or any other variables were significant risk factors. When the analysis was performed per procedure (Table 3), similar results were found showing MELD, Child-Pugh score and transfusion as risk factors for post-EBL bleeding. Patients that bled had higher Child-Pugh and MELD scores compared to those that did not bleed $(p=0.03$ and $p=0.02$, respectively). We selected the most relevant variables for the multivariate analysis per procedure and found that the MELD score was an independent risk factor for bleeding (Table 4).

All 26 patients with post-EBL bleeding were treated with vasoconstrictors (somatostatin/terlipressin), intravenous PPIs, and prophylactic antibiotics (norfloxacin or ceftriaxone) for 7 days. A gastroscopy was performed in all patients within 12-24 hours after admission to the hospital. Endoscopic therapy was performed with sclerotherapy in 6 and with band ligation in 6 patients. In the remaining cases no endoscopic therapy was performed. Two out of the 26 patients (7.7\%) (both Child-Pugh C class) died within 6 weeks due to multiorgan failure.

\section{Discussion}

In this analysis we evaluated the role of prophylactic administration of blood products in outpatients with cirrhosis undergoing EBL. To our knowledge this is the largest analysis evaluating the role of prophylactic transfusion of blood products in patients with cirrhosis and esophageal varices undergoing endoscopic band ligation. Our results demonstrated that the commonly used cut-offs for transfusion of FFP and platelets were only followed in $16 \%$ and $28 \%$ of procedures with high INR and/or low platelets, respectively. In addition, there was no association between INR or platelet count and bleeding events. These results reflect on the regular clinical practice for EBL in patients with cirrhosis in different hospitals and that current practice for all types of invasive procedures is not the same in all centers and is not equally adopted by all practitioners. For instance, current

\begin{tabular}{|c|c|c|c|c|c|c|}
\hline & & \multicolumn{2}{|l|}{ Bleeding } & \multicolumn{3}{|c|}{ Bleeding n, \% } \\
\hline & $\mathrm{n}$ & Yes & & Number & Yes $(n=26)$ & No $(n=510)$ \\
\hline \multirow{2}{*}{ INR $\geq 1.5$} & \multirow{2}{*}{108} & \multirow{2}{*}{$\begin{array}{l}\mathrm{n}=3 \\
2.7 \%\end{array}$} & FFP transfusion & $17(16 \%)$ & 1 & 16 \\
\hline & & & No FFP transfusion & $91(84 \%)$ & 2 & 89 \\
\hline \multirow{2}{*}{$\begin{array}{l}\text { Platelet } \\
\leq 50 \times 10^{9} / \mathrm{L}\end{array}$} & \multirow{2}{*}{85} & \multirow{2}{*}{$\begin{array}{c}n=6 \\
7 \%\end{array}$} & Platelet transfusion & $24(28 \%)$ & 4 & 20 \\
\hline & & & No platelet transfusion & $61(72 \%)$ & 2 & 59 \\
\hline
\end{tabular}

Fig. 1. Patients with INR $\geq 1.5$ and platelet counts $\leq 50,000 / \mu 1$ and the number of patients who bled and who underwent prophylactic transfusions. FFP, fresh frozen plasma; INR, international normalized ratio. 
Table 2. Univariate analysis of risk factors for bleeding after EBL in patients $(n=536)$ with cirrhosis.

\begin{tabular}{|c|c|c|c|}
\hline & Post-EBL bleeding $(n=26)$ & No post-EBL bleeding $(n=510)$ & $p$ value \\
\hline Age (years) & $61(49-68)$ & $61(52-70)$ & 0.55 \\
\hline $\operatorname{Sex}(M / F)$ & $20 / 6$ & $364 / 146$ & 0.65 \\
\hline Child-Pugh score & $7(6-8)$ & $6(5-8)$ & 0.07 \\
\hline Child-Pugh class A,B,C (\%) & 2.8/7.9/7.3 & 97.2/92.1/92.7 & $<0.03$ \\
\hline MELD score & $13(10-14)$ & $11(8-14)$ & $<0.02$ \\
\hline Etiology: HCV, alcohol, other (\%) & $(38 / 31 / 31)$ & $(41 / 30 / 29)$ & 0.84 \\
\hline Creatinine (mg/dl) & $0.93(0.7-1.2)$ & $0.8(0.7-1.1)$ & 0.25 \\
\hline Bilirubin (mg/dl) & $1.60(0.8-2.3)$ & $1.3(0.8-2.0)$ & 0.75 \\
\hline INR & $1.30(1.1-1.4)$ & $1.26(1.1-1.4)$ & 0.75 \\
\hline INR $\geq 1.5(\%)$ & $3(11)$ & $105(20)$ & \\
\hline Platelets $\times 10^{9} / \mathrm{L}$ & $73(53-112)$ & $91(60-126)$ & 0.27 \\
\hline Platelets $\leq 50 \times 10^{9} / \mathrm{L}(\%)$ & $6(23)$ & $79(15)$ & \\
\hline APTT (seconds) & $31(29-38)$ & $31(28-34)$ & 0.27 \\
\hline \multicolumn{4}{|l|}{ Transfusion, n (\%) } \\
\hline Any blood product & $5(19.2)$ & $32(6.3)$ & $<0.02$ \\
\hline FFP & $1(3.8)$ & $17(3.3)$ & 0.59 \\
\hline Platelets & $4(15.4)$ & $20(3.9)$ & $<0.02$ \\
\hline
\end{tabular}

APTT, activated partial thromboplastin time; EBL, endoscopic band ligation; FFP, fresh frozen plasma; INR, international normalized ratio; MELD, model for end-stage live disease. $p$ values in bold denote statistical significance. Data expressed as median (25-75\%) for continuous variables, or $n$ (\%) for categorical variables.

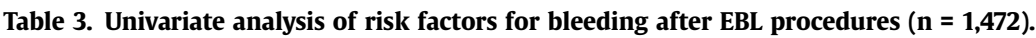

\begin{tabular}{|c|c|c|c|}
\hline & Post-EBL bleeding, $\mathrm{n}=33(2.2 \%)$ & No post-EBL bleeding $n=1,439(97.8 \%)$ & $p$ value \\
\hline Age (years) & $61(49-68)$ & $61(52-70)$ & .0 .79 \\
\hline Child-Pugh score & $7(6-8)$ & $6(5-8)$ & 0.07 \\
\hline Child-Pugh class A,B,C (\%) & $39 / 48 / 13$ & $67 / 28 / 5$ & $<0.001$ \\
\hline MELD score & $13(10-14)$ & $11(8-14)$ & 0.01 \\
\hline Etiology: HCV, alcohol, other (\%) & $(37 / 30 / 33)$ & $(39 / 28 / 33)$ & 0.84 \\
\hline Primary /secondary prophylaxis & $6 / 15(29 / 71 \%)$ & $497 / 656(43 / 57 \%)$ & 0.26 \\
\hline Creatinine $(\mathrm{mg} / \mathrm{dl})$ & $0.9(0.6-1.3)$ & $0.8(0.7-1.0)$ & 0.17 \\
\hline Bilirubin (mg/dl) & $1.3(0.7-2.3)$ & $1.2(0.8-1.8)$ & 0.78 \\
\hline INR & $1.3(1.2-1.4)$ & $1.23(1.1-1.4)$ & 0.10 \\
\hline INR $\geq 1.5(\%)$ & $5(15)$ & $243(16)$ & \\
\hline Platelets $\times 10^{9} / \mathrm{L}$ & $92(54-126)$ & $91(58-126)$ & 0.57 \\
\hline Platelets $\leq 50 \times 10^{9} / \mathrm{L}(\%)$ & $8(24)$ & $235(16)$ & \\
\hline APTT (seconds) & $33(29-38)$ & $30(28-33)$ & 0.05 \\
\hline Band session (first/repeated) & $5 / 16(24 / 76 \%)$ & $145 / 948(13 / 82 \%)$ & 0.20 \\
\hline \multicolumn{4}{|l|}{ Transfusion, n (\%) } \\
\hline Any blood product & $6(18.2)$ & $89(6.2)$ & 0.006 \\
\hline FFP & $2(6.1)$ & $28(1.9)$ & 0.09 \\
\hline Platelets & $5(15.2)$ & $74(5.1)$ & 0.01 \\
\hline
\end{tabular}

APTT, activated partial thromboplastin time; EBL, endoscopic band ligation; FFP, fresh frozen plasma; INR, international normalized ratio; MELD, model for end-stage liver disease. $p$ values in bold denote statistical significance. Data expressed as median (25-75\%) for continuous variables, or $\mathrm{n}$ (\%) for categorical variables.

Interventional Radiology guidelines recommend FFP transfusion for INR thresholds of less than 1.5-1.8 and 2.0-3.0 for high and low bleeding risk procedures, respectively ${ }^{16}$; that said, these thresholds are based upon expert opinion and are not validated by adequate clinical trials. The AGA and AASLD practice guidance state that these INR thresholds are not supported by evidence. ${ }^{10,17}$ In this regard, the results of this analysis are in line

Table 4. Multivariate analysis of risk factors for bleeding after EBL procedures $(n=1,472)$.

\begin{tabular}{lrrr}
\hline & OR & 95\% CI & $\boldsymbol{p}$ value \\
\hline MELD & 1.09 & $0.10-1.15$ & 0.006 \\
Etiology: HCV, alcohol, other (\%) & 1.25 & $0.79-1.98$ & 0.33 \\
Platelet count $\times 10^{9} / \mathrm{L}$ & 1.00 & $1.00-1.00$ & 0.83 \\
APTT (seconds) & 1.04 & $0.95-1.13$ & 0.34 \\
INR & 0.67 & $0.23-1.68$ & 0.40 \\
Transfusion pre-procedure & & & \\
$\quad$ FFP & 0.52 & $0.09-2.87$ & 0.45 \\
$\quad$ Platelets & 0.37 & $0.12-1.14$ & 0.08 \\
\hline
\end{tabular}

APTT, activated partial thromboplastin time; EBL, endoscopic band ligation; FFP, fresh frozen plasma; INR, international normalized ratio; OR, odds ratio; MELD, model for end-stage liver disease. with those of the Gastroenterology and Hepatology societies as we showed that there was no clear relationship between these test parameters and post-EBL bleeding..$^{10,17}$

Studies in the last decade indicate that chronic liver disease is not associated with an increased risk of bleeding. ${ }^{18-21}$ In fact, both pro- and anti-hemostatic pathways are altered and thus patients with cirrhosis are considered to have a rebalanced coagulation state; therefore, it is challenging to determine if there is an increased risk of bleeding or of developing thrombosis. ${ }^{18}$ Laboratory measures of coagulation in cirrhosis, such as the INR, prothrombin time or the activated partial thromboplastin time, are considered inadequate and in most cases can mislead the clinician because they only evaluate a portion of the hemostasis system and do not take into consideration other factors. The use of prophylaxis based on traditional measures of hemostasis prior to EBL is not recommended. ${ }^{10,18}$ Some experts state that platelet count values $<50,000 / \mu \mathrm{l}$ may be associated with higher risk of bleeding and platelet transfusion could be considered before high-risk procedures on a case-by-case basis. ${ }^{17}$ Current guidance suggests taking an individualized approach to patients with cirrhosis and thrombocytopenia prior to 
procedures owing to the lack of data on the safety and efficacy of transfusions. ${ }^{10}$ FFP is not recommended by any of the current guidelines or position papers since it does not reduce bleeding risk and may be associated with adverse events such as further volume expansion. ${ }^{12-14,10,17,18}$

The whole blood tests known as "viscoelastic tests" which include thromboelastography (TEG), rotational thromboelastometry (ROTEM) have been shown to be useful to guide transfusion in patients with cirrhosis that are actively bleeding during liver transplant and undergoing invasive procedures, but their ability to predict bleeding remains unknown. ${ }^{22,23}$ From a clinical standpoint, the main input of ROTEM/TEG in patients with liver disease is the negative predictive value. These tests are not routinely used in endoscopy units and we did not use them in our analysis. More information from prospective studies is needed to determine if these tests can predict bleeding after EBL in patients with cirrhosis.

Post-EBL bleeding occurred in 26 patients out of 536 patients (4.8\%) and in 33 out of 1,472 procedures (2.2\%), which is in keeping with previous reports. ${ }^{4-8}$ In most cases bleeding was due to post-EBL ulcer. An important finding in our series was that in those that bled after EBL, only 7 met criteria for transfusion and of these only 1 received FFP and 4 platelet transfusion; the remaining patients did not meet criteria for transfusion. There was no significant association between INR or platelet count and bleeding events on the multivariate analysis. That said, the univariate analysis (Table 2) did reveal that patients that received platelets had a higher tendency of bleeding compared with those that did not receive platelet transfusions (15\% vs. 4\%). Univariate and multivariate analysis revealed that Child-Pugh and MELD scores were risk factors for post-EBL bleeding. Of note, we performed the multivariate analysis with and without the INR, and the results remained unchanged. Etiology was included in the multivariate analysis because most of the patients had underlying HCV or alcohol. Taken together our findings also resonate with those of a recent meta-analysis that showed coagulation parameters like the INR fail to serve as a significant correlate for periprocedural bleeding events among patients with cirrhosis. $^{24}$

The current study has some limitations. First, it comprises a retrospective review of 4 centers and thus a prospective and longer assessment of the adverse events after EBL was not performed. Second, the study was not specifically designed to evaluate the role of prophylactic transfusion in patients with cirrhosis and EBL, rather it is an analysis and reflection of daily practice and the lack of data relating to endoscopic findings and operator experience is an important limitation. Thus, the use of prophylactic blood products was at the discretion of the clinicians and did not follow specific protocols or guidance. Third, the focus was on bleeding after EBL and other adverse events (i.e. anaphylactic reactions or lung injury after transfusion) were not recorded. Fourth, acute variceal bleeding was not evaluated and the reason for this was the heterogeneity of factors (vasoconstrictors, infections, intensive care unit stay, etc) that occur in this setting. Finally, although our results confirm data from other centers, ${ }^{7,8}$ these results require validation, although our analysis included 4 centers.

In conclusion, the results of this study confirm that the incidence of post-EBL bleeding and early bleeds is low and is associated with advanced liver disease; the commonly used cut-offs for transfusion of FFP and platelets were only followed in less than one-third of procedures; and, there was no association between INR or platelet count and bleeding events.

\section{Abbreviations}

EBL, endoscopic band ligation; FFP, fresh frozen plasma; INR, international normalized ratio; MELD, model for end-stage liver disease.

\section{Financial support}

Andrés Cárdenas is funded by the Instituto de Salud Carlos III and Plan Estatal de Investigación Ciéntifica y Técnica y de Innovación - Grant No PI19/ 00752 and has received funding for this work by "Fundación Marta Balust"

\section{Conflict of interest}

AC is a consultant for Mallinckrodt Pharmaceuticals, Boston Scientific Corp, Shionogi Inc, SOBI, B. Braun and has participated on Advisory Boards for Mallinckrodt Pharmaceuticals and SOBI and has received grant support by Mallinckrodt and Boston Scientific Corp.

Please refer to the accompanying ICMJE disclosure forms for further details.

\section{Authors' contributions}

A Blasi - acquired, analyzed, and interpreted the results, performed the statistical analysis, and drafted the manuscript. S Machlab - acquired, analyzed, and interpreted the results, performed the statistical analysis, and drafted the manuscript. R Risco - acquired, analyzed, and interpreted the results. JP Costa-Freixas- acquired, analyzed, and interpreted the results. G Hernandez-Cela - acquired, analyzed, and interpreted the results. D Horta- acquired, analyzed, and interpreted the results. A Bofill - acquired, analyzed, and interpreted the results. P Ruiz-Ramirez- acquired, analyzed, and interpreted the results. J Profitos - acquired data, analyzed, and interpreted the results. JM Sanahuja - interpreted the results and performed the statistical analysis. A Fernandez-Simon - acquired data, analyzed interpreted the results. M Vergara-Gómez - acquired, analyzed, and interpreted the results. J Sánchez-Delgado - participated in its design, interpreted the results, and drafted the manuscript. A Cárdenas - acquired, analyzed, and interpreted the results; conceived the study and participated in its design; performed the statistical analysis, drafted, edited, and revised the final manuscript.

\section{Data availability statement}

The raw data for this analysis is available all four participating hospitals. The content of the manuscript is the responsibility of the authors. All authors read, revised, and approved the final manuscript.

\section{Disclaimer}

Preliminary results of this study were presented at the Digital EASL International Liver Congress as an Oral Presentation on August 28, 2020.

\section{Supplementary data}

Supplementary data to this article can be found online at https://doi.org/1 0.1016/j.jhepr.2021.100363.

\section{References}

[1] Garcia-Tsao G, Abraldes JG, Berzigotti A, Bosch J. Portal hypertensive bleeding in cirrhosis: risk stratification, diagnosis, and management: 2016 practice guidance by the American Association for the study of liver diseases. Hepatology 2017;65:310-335.

[2] Tripathi D, Stanley AJ, Hayes PC, Patch D, Millson C, Mehrzad H, et al. U.K. guidelines on the management of variceal haemorrhage in cirrhotic patients. Gut 2015;64:1680-1704.

[3] Cárdenas A, Fernández-Simon A, Escorcell A. Endoscopic band ligation and esophageal stents for acute variceal bleeding. Clin Liver Dis 2014 Nov; 18(4):793-808. 


\section{Research article}

[4] Sinclair M, Vaughan R, Angus PW, Gow PJ, Parker F, Hey P, et al. Risk factors for band-induced ulcer bleeding after prophylactic and therapeutic endoscopic variceal band ligation. Eur J Gastroenterol Hepatol 2015 Aug;27(8):928-932.

[5] Dueñas E, Cachero A, Amador A, Rota R, Salord S, Gornals J, et al. Ulcer bleeding after band ligation of esophageal varices: risk factors and prognosis. Dig Liver Dis 2020 Jan;52(1):79-83.

[6] Vieira da Rocha EC, D’Amico EA, Caldwell SH, Flores da Rocha TR, Soares E, Silva CS, et al. A prospective study of conventional and expanded coagulation indices in predicting ulcer bleeding after variceal band ligation. Clin Gastroenterol Hepatol 2009 Sep;7(9):988-993.

[7] Drolz A, Schramm C, Seiz O, Groth S, Vettorazzi E, Horvatits T, et al. J. Risk factors associated with bleeding after prophylactic endoscopic variceal ligation in cirrhosis. Endoscopy 2021 Mar;53(3):226-234.

[8] Napolitano G, Iacobellis A, Merla A, Niro G, Valvano MR, Terracciano F, et al. Bleeding after invasive procedures is rare and unpredicted by platelet counts in cirrhotic patients with thrombocytopenia. Eur J Intern Med 2017 Mar;38:79-82.

[9] Funakoshi N, Duny Y, Valats JC, Ségalas-Largey F, Flori N, Bismuth M, et al. Meta-analysis: beta-blockers versus banding ligation for primary prophylaxis of esophageal variceal bleeding. Ann Hepatol 2012;11:369383.

[10] Northup PG, Garcia-Pagan JC, Garcia-Tsao G, Intagliata NM, Superina RA, Roberts LN, et al. Vascular liver disorders, portal vein thrombosis, and procedural bleeding in patients with liver disease: 2020 practice guidance by the American association for the study of liver diseases. Hepatology 2021 Jan;73(1):366-413.

[11] Slichter SJ. Relationship between platelet count and bleeding risk in thrombocytopenic patients. Transfus Med Rev 2004 Jul;18(3):153-156.

[12] Tripodi A, Chantarangkul V, Primignani M, Clerici M, Dell'era A, Aghemo A, et al. Thrombin generation in plasma from patients with cirrhosis supplemented with normal plasma: considerations on the efficacy of treatment with fresh-frozen plasma. Intern Emerg Med 2012 Apr;7(2):139-144.

[13] Rassi AB, d'Amico EA, Tripodi A, da Rocha TRF, Migita BY, Ferreira CM, et al. Fresh frozen plasma transfusion in patients with cirrhosis and coagulopathy: effect on conventional coagulation tests and thrombomodulin-modified thrombin generation. J Hepatol 2020 Jan;72(1):85-94.

[14] Bernal W, Caldwell SH, Lisman T. Nails in the coffin of fresh frozen plasma to prevent or treat bleeding in cirrhosis? J Hepatol 2020 Jan;72(1):12-13.

[15] de Franchis R, VI Faculty Baveno. Expanding consensus in portal hypertension: report of the Baveno VI Consensus Workshop: stratifying risk and individualizing care for portal hypertension. J Hepatol 2015 Sep;63(3):743-752.

[16] Patel IJ, Rahim S, Davidson JC, Hanks SE, Tam AL, Walker TG, et al. Society of interventional Radiology consensus guidelines for the periprocedural management of thrombotic and bleeding risk in patients undergoing percutaneous image-guided interventions-Part II: recommendations: endorsed by the Canadian association for interventional Radiology and the cardiovascular and interventional radiological society of europe J Vasc Interv Radiol 2019 Aug;30(8):1168-1184.e1.

[17] O'Leary JG, Greenberg CS, Patton HM, Caldwell SH. AGA clinical practice update: coagulation in cirrhosis. Gastroenterology 2019 Jul;157(1):3443.e1.

[18] Stine JG, Intagliata NM, Shah NL, Lisman T, Violi F, Caldwell SH, et al. Clinical cirrhosis dilemmas: survey of practice from the 7th international coagulation in liver disease conference. Dig Dis Sci 2020 May;65(5):13341339.

[19] von Meijenfeldt FA, van den Boom BP, Adelmeijer J, Roberts LN, Lisman T, Bernal W. Prophylactic fresh frozen plasma and platelet transfusion have a prothrombotic effect in patients with liver disease. J Thromb Haemost 2021 Mar;19(3):664-676.

[20] Wan J, Roberts LN, Hendrix W, Konings J, Ow TW, Rabinowich L, et al Whole blood thrombin generation profiles of patients with cirrhosis explored with a near patient assay. J Thromb Haemost 2020 Apr;18(4):834-843.

[21] Fisher C, Patel VC, Stoy SH, Singanayagam A, Adelmeijer J, Wendon J, et al. Balanced haemostasis with both hypo- and hyper-coagulable features in critically ill patients with acute-on-chronic-liver failure. J Crit Care 2018 Feb;43:54-60.

[22] De Pietri L, Bianchini M, Montalti R, De Maria N, Di Maira T, Begliomini B, et al. Thrombelastography-guided blood product use before invasive procedures in cirrhosis with severe coagulopathy: a randomized, controlled trial. Hepatology 2016;63(2):566-573.

[23] De Pietri L, Bianchini M, Rompianesi G, Bertellini E, Begliomini B. Thromboelastographic reference ranges for a cirrhotic patient population undergoing liver transplantation. World J Transpl 2016;6(3):583.

[24] Kovalic AJ, Majeed CN, Samji NS, Thuluvath PJ, Satapathy SK. Systematic review with meta-analysis: abnormalities in the international normalised ratio do not correlate with periprocedural bleeding events among patients with cirrhosis. Aliment Pharmacol Ther 2020 Oct;52(8):12981310. 